\title{
Representative Volume Element Homogenization of a Composite Material by Using Bond-based Peridynamics
}

\author{
Wenxuan Xia, Yakubu Kasimu Galadima, Erkan Oterkus and Selda Oterkus \\ Peridynamics Research Centre \\ Department of Naval Architecture, Ocean and Marine Engineering \\ University of Strathclyde, Glasgow United Kingdom
}

\begin{abstract}
This study presents representative volume element (RVE) homogenization by using bondbased peridynamics. First, a method to perform RVE homogenisation analysis with bond-based peridynamic theory is demonstrated. Homogenized material properties of a unit-sized fibre reinforced composite cell with varying fibre volume fraction are predicted using the present method. Peridynamic results are compared against a finite element based RVE approach and a good agreement is observed between the two approaches.
\end{abstract}

Keywords: Peridynamics; homogenisation; unit cell; composite material

\section{Introduction}

Representative Volume Element (RVE) analysis is a common micromechanics method in predicting effective material properties for heterogeneous materials such as composite materials. With extensive support provided by Finite Element Analysis (FEA), performing RVE analysis within the realm of FEA is straightforward and convenient to do. Recently, with the development of a new micromechanics method called Mechanics of Structure Genome (MSG) [1], software packages such as SwiftComp which utilize MSG become a feasible alternative when performing composite homogenization problems. However, since the majority of these micromechanics methods are based on Finite Element Method and Classical Continuum Mechanics, they encounter tremendous difficulties when dealing with damages such as cracks and voids. It is of interest to develop a new homogenization method that is not limited by the presence of discontinuities. Peridynamic (PD) theory [2] is particularly suited for this task since its governing equation is valid regardless of discontinuities.

PD theory is a new continuum mechanics formulation and has been applied for the analysis of many different material systems including metals [3] and composite materials [4,5]. PD theory is not limited to macro-scale analysis and has been used to analyse polycrystalline materials [6,7] and homogenisation [8]. PD theory can also be used for the analysis of other physical fields including thermal [9-11] and diffusion [12,13] fields. Impact problems can also be investigated by using PD theory [14]. PD theory can be utilised for modelling corrosion [15] and lithiation damage [16]. An extensive review of peridynamics is given in [17].

In this study, a particular type of PD theory, Bond-based Peridynamics, is used for Representative Volume Element (RVE) homogenization of a composite material by following an approach similar to Madenci et al. [8]. Several numerical cases are considered and peridynamic results are compared with analytical and Finite Element Analysis (FEA) results. 


\section{Bond-Based Peridynamic Theory}

Peridynamic (PD) theory introduced by Silling [2] is a nonlocal alternative to Classical Continuum Mechanics. PD theory is based on continuum assumption where a body is composed of infinitely small volumes called material points. In PD theory, material points are allowed to establish interaction (bond) with other material points within a finite range. This interaction range is defined as a PD parameter called "horizon". The motion of a material point is described by considering its interaction with all other points within its horizon. Therefore, PD theory replaces the partial differential operator existing in the governing equilibrium equation of Classical Continuum Mechanics with an integral operator. The integral domain in the PD governing equation is equal to the horizon of the material point. The horizon in PD theory can play a role of a bridge between PD theory and Classical Continuum Mechanics. The Classical Continuum Mechanics can be viewed as a limiting case of PD theory as the horizon size approaches to zero [18].

Bond-based PD theory, which can also be referred as original PD theory, considers the interaction between each pair of material points independently without considering the influence of other materials points inside the horizon. Therefore, the PD governing equation can be written as

$$
\rho \ddot{\mathbf{u}}(\mathbf{x}, t)=\int_{H} \mathbf{f}\left(\mathbf{u}^{\prime}-\mathbf{u}, \mathbf{x}^{\prime}-\mathbf{x}, t\right) d V^{\prime}+\mathbf{b}(\mathbf{x}, t)
$$

where $\rho$ is the density, $t$ is time, $H$ is horizon, $\ddot{\mathbf{u}}$ and $\mathbf{u}$ are the acceleration and displacement of the material point located at $\mathbf{x}$, and $\mathbf{b}$ is the body load density. Peridynamic force between the material points located at $\mathbf{x}$ and $\mathbf{x}^{\prime}$ can be expressed as

$$
\mathbf{f}=\operatorname{css} \frac{\mathbf{x}^{\prime}-\mathbf{x}+\mathbf{u}^{\prime}-\mathbf{u}}{\left|\mathbf{x}^{\prime}-\mathbf{x}+\mathbf{u}^{\prime}-\mathbf{u}\right|}
$$

where $c$ is the bond constant and the stretch, $s$ can be defined as

$$
S=\frac{\left|\mathbf{x}^{\prime}-\mathbf{x}+\mathbf{u}^{\prime}-\mathbf{u}\right|-\left|\mathbf{x}^{\prime}-\mathbf{x}\right|}{\left|\mathbf{x}^{\prime}-\mathbf{x}\right|}
$$

The bond constant, $c$ can be related to the material constants of Classical Continuum Mechanics for two-dimensional configurations as $c=9 E /\left(\pi h \delta^{3}\right)$ with $E, \delta$ and $h$ being the Elastic modulus, horizon size and thickness, respectively. 


\section{Representative Volume Element Homogenization in Bond-Based Peridynamics}

Similar to performing RVE analysis in FEA, the implementation of RVE analysis in PD theory can be summarised into the following steps:

1. Selection of RVE

2. Numerical modelling of the cell

3. Apply carefully crafted boundary conditions and solve certain sets of boundary value problems

4. Process the evaluated stress and strain fields to obtain desired effective properties

\section{Boundary conditions}

Common boundary conditions (BC) for RVE analysis can be categorized into homogeneous $\mathrm{BC}$ and periodic $\mathrm{BC}$. Homogeneous $\mathrm{BC}$ includes homogeneous displacement boundary condition and homogeneous stress boundary condition. Homogeneous displacement $\mathrm{BC}$ can be written as

$$
u_{i}^{s}=\bar{\varepsilon}_{i j} x_{j}
$$

where the strain $\bar{\varepsilon}_{i j}$ is homogeneous and therefore constant along the RVE boundary surface. $x_{j}$ is the relative distance between the two opposite sides of RVE. Similar to homogeneous displacement boundary condition, the homogeneous stress boundary condition can be written as

$$
t_{i}^{S}=\bar{\sigma}_{i j} n_{j}
$$

where the stress $\bar{\sigma}_{i j}$ is homogeneous and therefore constant along the RVE boundary surface. $n_{j}$ is the unit normal to the RVE boundary surface. When being applied to RVE homogenization, Eqs. (4) and (5) will create a homogeneous stress or strain field inside the RVE.

The other type of $\mathrm{BC}$, named as periodic $\mathrm{BC}$, can be stated as

$$
t_{i}^{+}-t_{i}^{-}=0, u_{i}^{+}=u_{i}^{-}+\bar{\varepsilon}_{i j}\left(x_{j}^{+}-x_{j}^{-}\right)
$$

in which the superscript represents the corresponding surface pairs. Similar to the most displacement based FEA RVE packages, only the displacement part of the periodic BC needs to be applied and the traction part of the periodic $\mathrm{BC}$ will be automatically satisfied as long as the discretization is fine enough and the resulted stress field is converged.

Normally, the size of a RVE need to be large enough so that the properties of the RVE are statistically identical to the macroscopic material in whole. However, in practice, the minimum RVE size to reach statistically identical condition is not always obvious. It has been proven that homogeneous displacement boundary condition and homogeneous stress boundary condition create upper and lower bounds for the effective material properties, respectively. When the size of the RVE increases, the effective material properties obtained from homogeneous BC will eventually converge to those obtained from periodic BC [19]. Therefore, although it is entirely feasible to apply homogeneous BC in Bond-based Peridynamics (BBPD), the more accurate periodic BC is adopted in the present study. In BBPD RVE method, Eq. (6) 
is applied to BBPD governing equations using the technique of Lagrange multipliers. Several fictitious regions are created surrounding the RVE upon which the BCs are applied.

\section{Effective material properties}

All BCs in RVE homogenization serve the same purpose as to obtain the effective material properties matrix $C^{*}$ or effective compliance $S^{*}$ from the constitutive relations given in Eqs. (7) and (8) by satisfying the Hill's macrohomogeneity condition [20] which states that the volume average of stress $\left\langle\sigma_{k l}\right\rangle$ or strain $\left\langle\varepsilon_{k l}>\right.$ of the RVE can be determined by the average stress $\bar{\sigma}_{i j}$ or strain $\bar{\varepsilon}_{i j}$ at RVE surface $S$.

$$
\begin{aligned}
& <\sigma_{i j}>=C_{i j k l}^{*} \cdot<\varepsilon_{k l}> \\
& <\varepsilon_{i j}>=S_{i j k l}^{*} \cdot<\sigma_{k l}>
\end{aligned}
$$

In the constitutive relations given in Eqs. (7) and (8), once the volume average of strain or stress field on the right hand side is determined to be constant through Hill's condition, the effective material property $C_{i j k l}^{*}$ and its compliance $S_{i j k l}^{*}$ can be obtained by processing the resulted stress or strain field on the left hand side of the equation. 


\section{Numerical Results}

The present BBPD RVE method is validated by comparing the effective material properties of the unit cell obtained from BBPD with either analytical solution or FEA based homogenization approach named as Mechanics of Structure Genome (MSG) developed by Yu in 2015 [1]. The MSG analysis is performed using SwiftComp software [21].

The first example considers a simple unit length homogeneous isotropic cell without any fibre. The Young's modulus of the constituent material is $E=68.3 \mathrm{GPa}$ and Poison's ratio is $1 / 3$. The effective material property matrix of the cell is then determined using BBPD RVE as

$$
C^{*}=\left[\begin{array}{ccc}
76.8375 & 25.6125 & 0 \\
25.6125 & 76.8375 & 0 \\
0 & 0 & 25.6125
\end{array}\right] \mathrm{GPa}
$$

The evaluated effective Young's modulus is $68.3 \mathrm{GPa}$, the effective Poisson's ratio is $1 / 3$ and the effective shear modulus is $25.6125 \mathrm{GPa}$. The computed effective material properties are all identical to the analytical solution done through plane stress assumption.

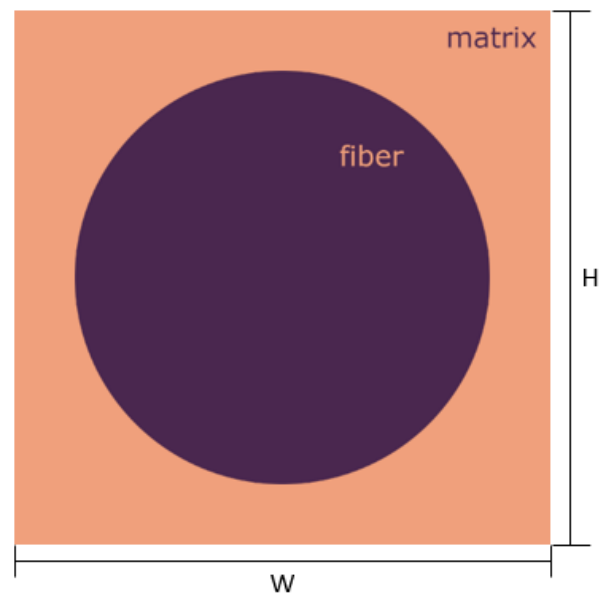

Figure 1. Two dimensional fibre reinforced composite cell

The second example considers a cell with centrally positioned fibre inclusion as shown in Fig. 1. The dimensions $W$ and $H$ of the cell are specified as 1 . The geometry is discretized into 50 by 50 grids with 2,500 material points and 5,000 degrees of freedom. The constituent materials of both fibre and matrix are isotropic. Centrally positioned fibre possesses a Young's modulus of $E_{f}=379.3 \mathrm{GPa}$ while the surrounding matrix has a Young's modulus of $E_{m}=$ 68.3 GPa. The Poisson's ratio for both fibre and matrix are equal to $1 / 3$. Four different cases with varying fibre volume fraction ranging from $10 \%$ to $40 \%$ are considered, with an interval of $10 \%$ (see Table 1 for the list of fibre volume fraction cases) 
Table 1. List of fibre volume fraction cases

\begin{tabular}{|r|l|}
\hline \multicolumn{2}{|c|}{$\begin{array}{c}\text { Square Pack } \\
\text { Microstructure }\end{array}$} \\
\hline $\begin{array}{c}\text { Volume } \\
\text { fraction }\end{array}$ & Fibre radius \\
\hline 0.1 & 0.178412412 \\
0.2 & 0.252313252 \\
0.3 & 0.309019362 \\
0.4 & 0.356824823 \\
\hline
\end{tabular}

Employing the BBPD RVE approach, the effective material property matrices under two dimensional plane stress assumption are determined as

$$
\begin{gathered}
C_{v f=0.1}^{*}=\left[\begin{array}{ccc}
87.7401 & 28.6991 & 0 \\
28.6991 & 87.7401 & 0 \\
0 & 0 & 29.0392
\end{array}\right] \mathrm{GPa} \\
C_{v f=0.2}^{*}=\left[\begin{array}{ccc}
102.2417 & 32.3705 & 0 \\
32.3705 & 102.2417 & 0 \\
0 & 0 & 33.3731
\end{array}\right] \mathrm{GPa} \\
C_{v f=0.3}^{*}=\left[\begin{array}{ccc}
119.5032 & 36.3852 & 0 \\
36.3852 & 119.5032 & 0 \\
0 & 0 & 38.3356
\end{array}\right] \mathrm{GPa} \\
C_{v f=0.4}^{*}=\left[\begin{array}{ccc}
137.7930 & 40.0253 & 0 \\
40.0253 & 137.7930 & 0 \\
0 & 0 & 43.0530
\end{array}\right] \mathrm{GPa}
\end{gathered}
$$

The results show that the effective material properties of the cell are transversely isotropic as expected from this configuration. The BBPD RVE predictions for the effective Young's modulus $E^{*}$ for different fibre volume fractions are shown in Figure 2. Moreover, the effective shear modulus $G^{*}$ for different fibre volume fractions are presented in Figure 3 . The BBPD RVE predictions are compared with homogenization solutions obtained from SwiftComp software. It is observed that a slight deviation occurs in both effective properties of Young's modulus and shear modulus when the fibre volume fraction increases. The maximum deviation for effective Young's modulus is below 4\%. The maximum deviation for effective shear modulus is $5.24 \%$. Results of both $E^{*}$ and $G^{*}$ are in acceptable agreement for all fibre volume fractions cases as shown in Table 2 . 


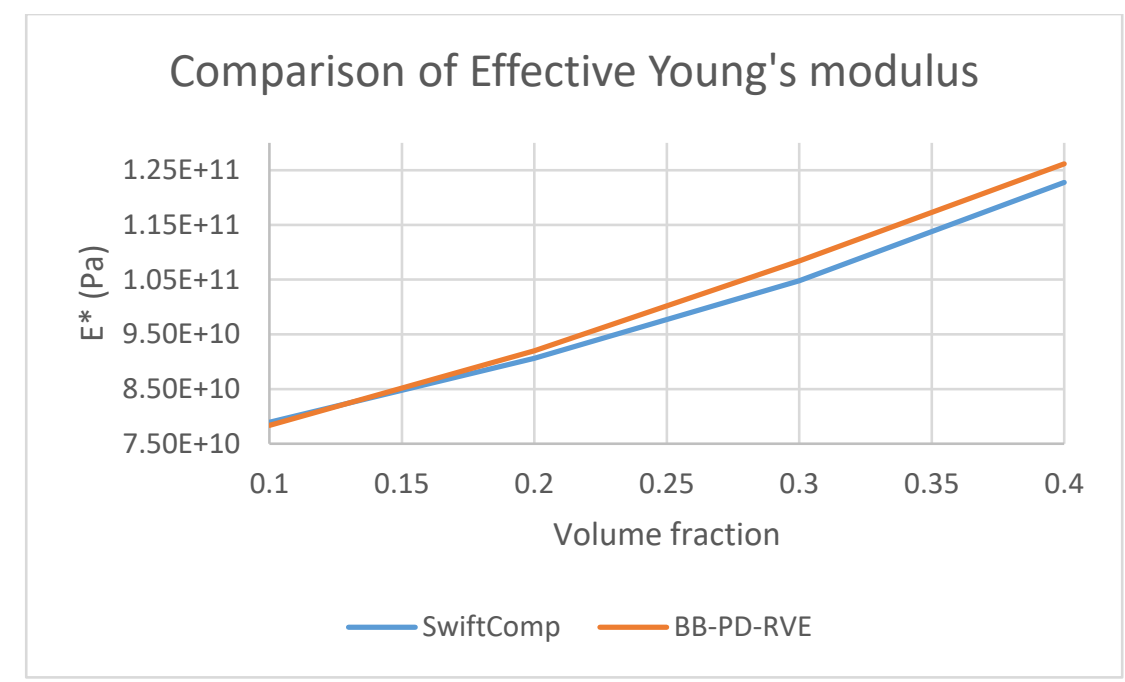

Figure 2. Comparison of effective Young's modulus

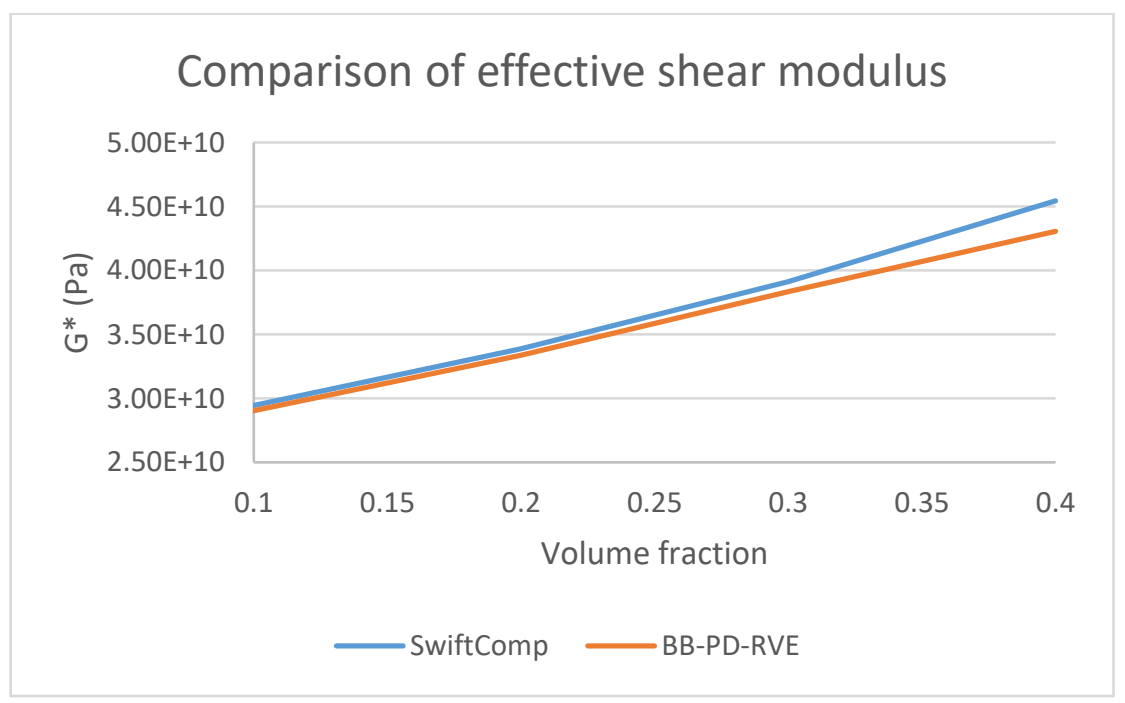

Figure 3. Comparison of effective shear modulus

Table 2. Comparison of the BBPD RVE predictions for material constants with MSG

\begin{tabular}{|c|c|c|c|c|c|c|}
\hline & \multicolumn{2}{|c|}{ SwiftComp } & \multicolumn{2}{c|}{ BBPD RVE } & \multicolumn{2}{c|}{ Difference } \\
\hline $\begin{array}{c}\text { Volume } \\
\text { fraction }\end{array}$ & $\mathrm{E}^{*}$ & $\mathrm{G}^{*}$ & $\mathrm{E}^{*}$ & $\mathrm{G}^{*}$ & $\mathrm{E}^{*}$ & $\mathrm{G}^{*}$ \\
\hline 0.1 & $7.89 \mathrm{E}+10$ & $2.94 \mathrm{E}+10$ & $7.84 \mathrm{E}+10$ & $2.90 \mathrm{E}+10$ & $-0.73 \%$ & $-1.38 \%$ \\
0.2 & $9.06 \mathrm{E}+10$ & $3.39 \mathrm{E}+10$ & $9.20 \mathrm{E}+10$ & $3.34 \mathrm{E}+10$ & $1.51 \%$ & $-1.52 \%$ \\
0.3 & $1.05 \mathrm{E}+11$ & $3.91 \mathrm{E}+10$ & $1.08 \mathrm{E}+11$ & $3.83 \mathrm{E}+10$ & $3.45 \%$ & $-2.02 \%$ \\
0.4 & $1.23 \mathrm{E}+11$ & $4.54 \mathrm{E}+10$ & $1.26 \mathrm{E}+11$ & $4.31 \mathrm{E}+10$ & $2.77 \%$ & $-5.24 \%$ \\
\hline
\end{tabular}




\section{Conclusions}

In this study, Bond-based Peridynamics is used to perform representative volume element homogenization of a composite material. Numerical solutions show that the present BBPD RVE approach is capable of performing homogenization for a composite cell with random fibre volume fractions at a similar level of effectiveness as other FEA based RVE analysis approaches in terms of predicting effective Young's modulus and shear modulus. It also opens the possibility of performing damage analysis onto the RVE cell by simply breaking PD bonds across the damage surface. 


\section{References}

[1] Yu, W., 2015. Structure genome: fill the gap between materials genome and structural analysis. In 56th AIAA/ASCE/AHS/ASC Structures, Structural Dynamics, and Materials Conference (p. 0201).

[2] Silling, S.A., 2000. Reformulation of elasticity theory for discontinuities and long-range forces. Journal of the Mechanics and Physics of Solids, 48(1), pp.175-209.

[3] Madenci, E. and Oterkus, S., 2016. Ordinary state-based peridynamics for plastic deformation according to von Mises yield criteria with isotropic hardening. Journal of the Mechanics and Physics of Solids, 86, pp.192-219.

[4] Oterkus, E. and Madenci, E., 2012. Peridynamic theory for damage initiation and growth in composite laminate. In Key Engineering Materials (Vol. 488, pp. 355-358). Trans Tech Publications.

[5] Oterkus, E. and Madenci, E., 2012. Peridynamics for failure prediction in composites. In 53rd AIAA/ASME/ASCE/AHS/ASC Structures, Structural Dynamics and Materials Conference 20th AIAA/ASME/AHS Adaptive Structures Conference 14th AIAA (p. 1692).

[6] De Meo, D., Zhu, N. and Oterkus, E., 2016. Peridynamic modeling of granular fracture in polycrystalline materials. Journal of Engineering Materials and Technology, 138(4), p.041008.

[7] Zhu, N., De Meo, D. and Oterkus, E., 2016. Modelling of granular fracture in polycrystalline materials using ordinary state-based peridynamics. Materials, 9(12), p.977.

[8] Madenci, E., Barut, A. and Phan, N., 2018. Peridynamic unit cell homogenization for thermoelastic properties of heterogenous microstructures with defects. Composite Structures, 188, pp.104-115.

[9] Oterkus, S. and Madenci, E., 2014. Peridynamics for fully coupled thermomechanical analysis of fiber reinforced laminates. In 55th AIAA/ASMe/ASCE/AHS/SC Structures, Structural Dynamics, and Materials Conference (p. 0694).

[10] Alpay, S. and Madenci, E., 2013. Crack growth prediction in fully-coupled thermal and deformation fields using peridynamic theory. In 54th AIAA/ASME/ASCE/AHS/ASC structures, structural dynamics, and materials conference (p. 1477).

[11] Gao, Y. and Oterkus, S., 2019. Ordinary state-based peridynamic modelling for fully coupled thermoelastic problems. Continuum Mechanics and Thermodynamics, 31(4), pp.907937.

[12] Diyaroglu, C., Oterkus, S., Oterkus, E. and Madenci, E., 2017. Peridynamic modeling of diffusion by using finite-element analysis. IEEE Transactions on Components, Packaging and Manufacturing Technology, 7(11), pp.1823-1831.

[13] Diyaroglu, C., Oterkus, S., Oterkus, E., Madenci, E., Han, S. and Hwang, Y., 2017. Peridynamic wetness approach for moisture concentration analysis in electronic packages. Microelectronics Reliability, 70, pp.103-111. 
[14] Oterkus, E., Guven, I. and Madenci, E., 2012. Impact damage assessment by using peridynamic theory. Open Engineering, 2(4), pp.523-531.

[15] De Meo, D. and Oterkus, E., 2017. Finite element implementation of a peridynamic pitting corrosion damage model. Ocean Engineering, 135, pp.76-83.

[16] Wang, H., Oterkus, E. and Oterkus, S., 2018. Predicting fracture evolution during lithiation process using peridynamics. Engineering Fracture Mechanics, 192, pp.176-191.

[17] Javili, A., Morasata, R., Oterkus, E. and Oterkus, S., 2019. Peridynamics review. Mathematics and Mechanics of Solids, 24(11), pp.3714-3739.

[18] Madenci, E. and Oterkus, E., 2014. Peridynamic Theory. In Peridynamic Theory and Its Applications (pp. 19-43). Springer, New York, NY.

[19] Drago, A. and Pindera, M.J., 2007. Micro-macromechanical analysis of heterogeneous materials: macroscopically homogeneous vs periodic microstructures. Composites science and technology, 67(6), pp.1243-1263.

[20] Hill, R., 1972. On constitutive macro-variables for heterogeneous solids at finite strain. Proceedings of the Royal Society of London. A. Mathematical and Physical Sciences, 326(1565), pp.131-147.

[21] Yu, W. and Liu, X., 2015. SwiftComp. 